\title{
Afrontar el tratamiento de la Enfermedad Renal Crónica
}

\author{
Claudia Andrea Ramírez-Perdomo \\ ${ }^{1}$ Enfermera. Especialista en Cuidado crítico, Magíster en Enfermería. Doctora en Enfermería. Docente Facultad de \\ Salud. Universidad Surcolombiana. Neiva-Huila. Colombia
}

Como citar este artículo:

Ramírez-Perdomo CA. Afrontar el tratamiento dela Enfermedad Renal Crónica.

Enferm Nefrol. 2019 0ct-Dic;22(4):379-87

\section{Resumen}

Objetivo: Explorar de manera comprensiva la experiencia vivida en el tratamiento de la Enfermedad Renal Crónica y los significados del cuidado elaborados por enfermeras y familiares que interactúan con estas personas.

Material y Método: Estudio cualitativo, enfoque fenomenológico-hermenéutico, empleando el referente teórico de Van Manen, 11 personas con la enfermedad, 5 cuidadores y 5 enfermeros participaron en el estudio, en la ciudad de Neiva; información recolectada a través de entrevistas en profundidad.

Resultados: emergió el dilema entre la libertad y la vida al construir el sentido de afrontar el tratamiento de la Enfermedad Renal Crónica, los subtemas se enmarcaron en la dureza de la hemodiálisis, el significado de la máquina de diálisis, la apariencia de la fístula arterio venosa, el impacto del catéter peritoneal y la dieta.

Conclusiones: el tratamiento de la enfermedad irrumpe en la vida de las personas produciendo cambios abruptos en su vida que los lleva a reconocer la finitud de su existencia, las alternativas para sobrevivir, los cambios en su corporeidad. De los cuales no son ajenos sus cuidadores y enfermeras, llamados a brindarles apoyo para lograr enfrentar el tratamiento.

PALABRAS CLAVES: enfermedad renal crónica; , hemodiálisis; diálisis peritoneal; enfermería; cuidadores; investigación cualitativa; hermenéutica.

\section{Correspondencia:}

Claudia Andrea Ramírez Perdomo

E-mail: clauram1@hotmail.com

\section{Coping treatment in Chronic kidney disease}

\section{Abstract}

Aim: to explore in a comprehensive way the experience lived in the treatment of the Chronic Kidney Disease and the meanings of the care elaborated by nurses and relatives which interact with these people.

Material and Method: Qualitative study, phenomenology hermeneutic approach, using the theory concerning of Van Manen, 11 people with the disease, 5 caregivers and 5 nurses participated on the study, at the city of Neiva, the information was recollected by in-depth interviews.

Results: The dilemma between life and liberty when building the sense of dealing with the treatment of Chronic Kidney Disease, the subtopics were framed in the hardness of hemodialysis, the meaning of the dialysis machine, the appearance of arteriovenous fistula, the impact of the peritoneal catheter and diet.

Conclusions: the treatment of the disease bursts in the life of people producing abrupt changes in their life which leads them to recognize the finitude of their existence, the alternatives for surviving, the changes in their corporeity. Which are no outsiders to their caregivers and nurses called to give them support in order to face the treatment.

KEYWORDS: renal insufficiency; chronic; hemodialysis; peritoneal dialysis; nursing; caregivers; qualitative research; hermeneutics.

\section{Introducción}

A nivel mundial, el número de pacientes con Enfermedad Renal Crónica (ERC) ha aumentado de manera 
exponencial ${ }^{1}$, convirtiéndose en un importante problema de salud pública dada sus altas tasas de incidencia, morbilidad, mortalidad y sobrecarga de los sistemas de salud ${ }^{2}$, que amenaza con alcanzar proporciones epidémicas en las próximas décadas. Es una enfermedad crónica, debilitante, en la cual se pierde de manera gradual y progresiva la función renal2,3; es predictor de la disminución de la calidad de vida que afecta la persona y su familia de diferentes maneras ${ }^{4,5}$.

Existen varios factores importantes en su desarrollo, el envejecimiento, las enfermedades cardiovasculares y la diabetes mellitus tipo II; responsables de la creciente incidencia en adultos ${ }^{6}$. Después del diagnóstico comienzan su Terapia de Reemplazo Renal (TRR) para controlar los síntomas urémicos y mejorar sus condiciones de vida. Pueden ingresar a Hemodiálisis (HD) o Diálisis Peritoneal (DP) e iniciar el proceso del trasplante renal.

Es por ello, que recibir el diagnóstico y tener que asumir un proceso de modificación radical del estilo de vida, impacta profundamente a las personas afectadas, lo que incidirá en su calidad de vida y en las posibilidades de éxito de las intervenciones a las que se someta.

Lo anteriormente descrito, permitió plantear como objetivos:

1) Reconstruir el significado de la experiencia vivida con la Enfermedad Renal Crónica y el tratamiento dialítico, para las personas enfermas.

2) Develar los significados que sobre el tratamiento dialítico han elaborado los familiares que interactúan de forma más cercana con las personas afectadas por la enfermedad.

3) Recuperar los significados elaboradas por las enfermeras en los servicios diálisis acerca de cómo cuidar personas afectadas por la Enfermedad Renal Crónica.

\section{Materiales y Método}

Investigación cualitativa con enfoque fenomenológico-hermenéutico, basado en la propuesta de Van Ma$n n^{7}$. Se realizaron trece entrevistas a once personas con ERC en la ciudad de Neiva, 6 mujeres y 5 hombres, edad promedio 45 años, escolaridad variada, 7 estuvieron en tratamiento de HD y DP, 3 en tratamiento de HD y 1 en DP. Los cuidadores familiares fueron 4 mujeres y 1 hombre; edad promedio 45 años, escolaridad diversa, relación con las personas con ERC esposa/o y madre, con ocupación diversa. Por otro lado, los profesionales de Enfermería 4 mujeres y 1 hombre fueron entrevistados, edad promedio 52,6 años, tiempo promedio de labor en la unidad renal de 22,2 años.

Para identificar a los participantes se les asignó un código que correspondía a las iniciales de sus nombres, con el objetivo de diferenciar los grupos se colocó como letra inicial $P$ (persona con ERC), C (cuidador) y $E$ (Profesionales de Enfermería). Se empleó el muestreo intencional orientado por los criterios de inclusión, lo cual permitía asegurar la calidad de los datos ${ }^{8}$.

Los criterios de inclusión para las personas con ERC: mayor de 18 años, haber estado en tratamiento de HD o DP. Para los cuidadores: mayor de 18 años, cuidador familiar principal significativo en el proceso de cuidado. Para las enfermeras: estar o haber estado vinculada laboralmente con una unidad renal o de trasplante por un período mayor a 6 meses, significativo dentro de la experiencia con la enfermedad.

Sustentadas en las experiencias personales y profesionales en los servicios de diálisis, los conocimientos y el interés por el fenómeno de estudio, se solicitó a la Unidad de trasplante Surcolombiana la base de datos con la información de las personas. La información fue recolectada durante el 2017, mediante la entrevista en profundidad; la investigadora se puso en contacto con los participantes por vía telefónica y estos fueron entrevistados en su casa, partiendo de la primera entrevista se continuo con el proceso de recolección de la información de los participantes; la participación de estos fue voluntaria. Se partió de una pregunta general; para las personas enfermas: ¿Cómo ha sido su experiencia con el tratamiento dialítico de la ERC? Para los cuidadores familiares: ¿Qué ha significado tener que cuidar a su familiar en el tratamiento dialítico para la ERC? Para las enfermeras ¿Cómo es la experiencia de cuidar una persona con ERC en el tratamiento de diálisis? Las entrevistas duraron de 60 a 90 minutos, grabadas, escuchadas y transcritas de manera textual por la investigadora.

El análisis se realizó siguiendo la propuesta de Van Manen $\left.{ }^{7}: 1\right)$ recolección de la experiencia vivida, 2) reflexión de la experiencia vivida, 3 ) lectura y relectura de las entrevistas, 4) recuperar el lenguaje semántico dentro del texto, 5) detección de temas emergentes, y 
6) escritura y re-escritura de la experiencia vivida. Avalado por el Comité de Ética de la Universidad de Antioquía, Acta No CEI-FE2015-05. Se tuvo en cuenta la Resolución 8430 de 1993, principios éticos de beneficencia, autonomía, privacidad, libertad de expresión y sentimientos; y los siguientes criterios de rigor?: credibilidad y confirmabilidad mediante transcripción textual de las entrevistas por parte de la investigadora, los resultados fueron revisados por dos expertos en investigación cualitativa y posteriormente devueltas a los participantes. La recolección de la información finalizó una vez se consideró que se había obtenido suficiente información, que permitiera construir textos fenomenológicos enriquecidos. Con la transferibilidad, se espera que los resultados sirvan para encontrar similitudes 0 divergencias con otras investigaciones que aborden el fenómeno.

\section{Resultados}

En el presente artículo se analiza y discute el tema "el dilema entre la libertad y la vida al construir el sentido de afrontar el tratamiento de la ERC" y los subtemas derivados (Figura 1), como se presenta a continuación, en los tres grupos indagados.

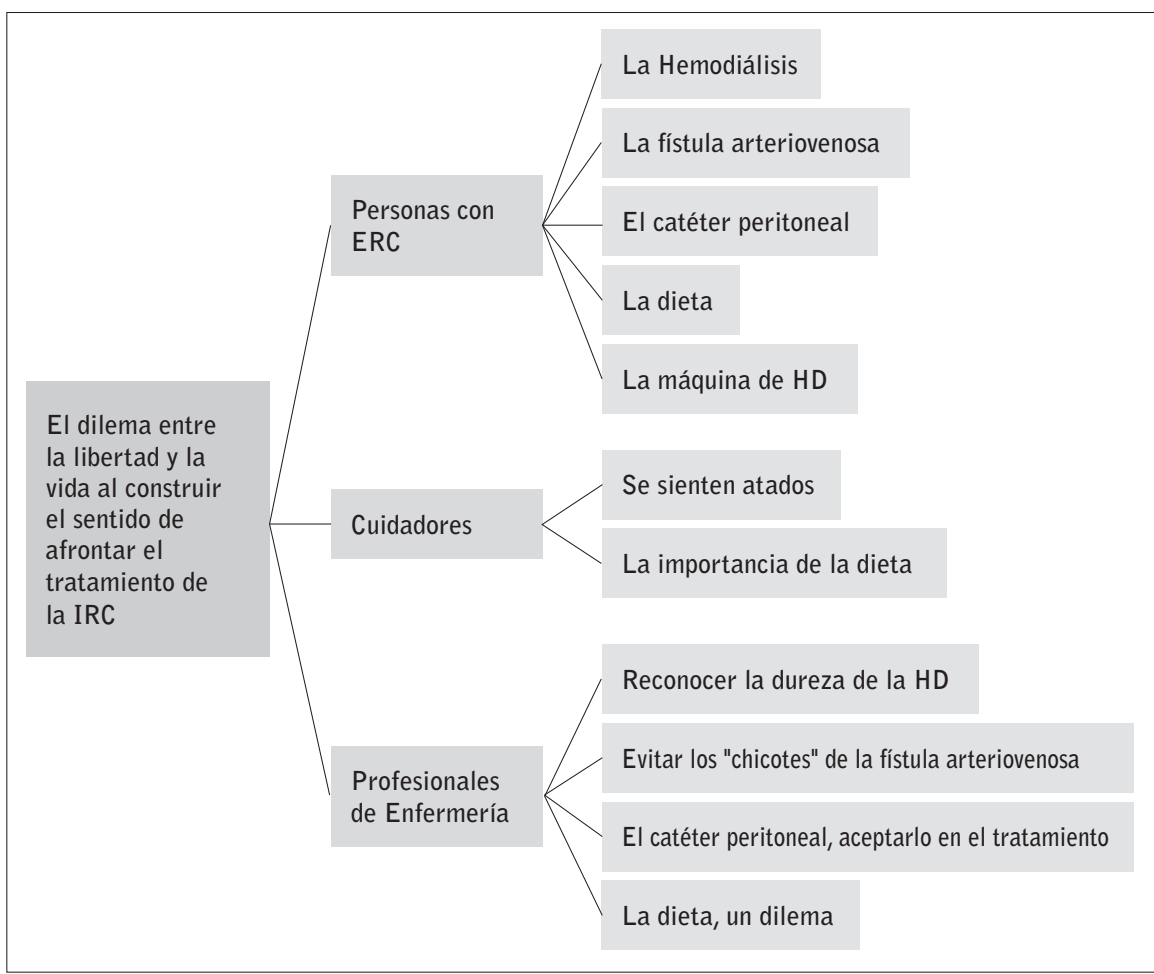

Figura 1. Subtemas emergentes organizados en los grupos de personas con ERC, cuidadores y Profesionales de Enfermería.

\section{Personas con ERC los subtemas reportados son:}

La HD: la ambivalencia ente la sobrevivencia y la muerte. En la mayoría de los casos, las personas con ERC consideran el tratamiento como un procedimiento muy duro. Durante la HD presentan complicaciones que los Ilevan a reconocer la dureza del tratamiento. La falta de apoyo de la familia los hace acudir al tratamiento solos, esto es difícil porque al salir descompensados del tratamiento deben enfrentar solos esta situación, esperando en algún rincón de la calle que el malestar les pase. Se consideran afortunados cuando cambian de residencia debido a que la situación toma otro rumbo.

La HD es dura, cada vez que me la hacían me daba dolor en la cintura, me hacía vomitar... era duro. En Bogotá era complicado porque eso era plata y muchas veces no tenía quien me acompañara, salía malita, en la calle me daba mareo, tenía que sentarme en un andén a esperar que me pasara, allá todo queda lejos, gracias a Dios me vine a vivir a Neiva...(P-VG)

La experiencia de la HD es angustiante, descrita como una competencia psicológica y emocional, una lucha constante contra la muerte. Al ingresar al tratamiento perciben que se enfrentan a la muerte, esa cercanía hace que al salir del tratamiento sientan que ganaron la batalla contra esta. Un ejercicio traumático y duro, al que tienen que asistir de manera interdiaria, donde se puede perder la vida en un instante.

En HD era angustiosa, una competencia psicológica, emocional, como si hiciéramos una lucha contra la muerte, la persona que iba saliendo como, "salí, ya terminé" (Ilanto), era angustioso cuando uno se quedaba sola, porque sabía que había riesgo de morirse en ese momento... una lucha entre la vida y la muerte, se pierde la vida en un instante... (P-MAA)

"Las montañitas" de la fístula arteriovenosa. La fístula asusta no solo a la persona enferma sino también a los que se encuentran cerca, los brazos se deforman describiéndolos como 
una "culebra" y "montañitas" las ocultan debido a que son objeto de la curiosidad de otros. En este mismo sentido, sugieren a las otras personas enfermas que se apliquen cremas para desvanecer los chichotes, los hombres no le dan importancia, mientras que las mujeres las ocultan de la mirada de los demás.

La fístula me asustaba, a esos señores se les ponía el brazo como una culebra. Apenas me sacaban de HD me ponía y sóbeme para que no se me brotara, arriba tenía montañitas, de tanto sobarme se desvanecieron. Me daba pena ver esas montañas, se ve feo. Los hombres decían eso no es nada y las mujeres las ocultan con una blusa de manga larga. (P-MR)

El catéter peritoneal "una manguera que cuelga". Sentir que su cuerpo se deforma a causa del catéter, despierta enojo. Al mirarse al espejo descubren el catéter colgando esto les parece fuerte, llevándola a cuestionarse en qué momento ha pasado esto. Sin embargo, el acompañamiento de su pareja es vital para reconocer como ese tubito le permite estar viva. Aprende a valorar la importancia del catéter, como un vínculo con la vida, desaparece la rabia experimentada por el tratamiento.

Al comienzo da rabia con el catéter, deforme mi cuerpo es lo que uno piensa, con el tiempo ya no me daba rabia, además, jugábamos con el (risas), me decía "gracias a lo que hace a través de ese tubito está viva". Hay momentos en que se ve esa cosa colgando que llegaba hasta la rodilla. Entendí que gracias a ese tubito estaba viva y aprendí a valorarlo. (P-MCV)

La dieta: la encrucijada entre cuidarse o morir de hambre. La dieta es otro aspecto difícil de afrontar, las indicaciones de las nutricionistas no son claras, por lo tanto, no logran acomodarse a esta. Pierden peso y eso los obliga a replantear el seguimiento de la misma. Se cuidan del consumo de sal. Están inconformes con el manejo de la nutricionista, para ellos este profesional les recomienda dejar de consumir los alimentos que le gustan, y dichas recomendaciones son muy costosas e imposibles de proseguir. El consumo desmedido de agua, los lleva a la aparición de edemas y fatiga; situación que los obliga a intentar durante el tratamiento dialítico bajar el sobrepeso alcanzado para recuperar su peso ideal.

Nos decían que dializáramos las papas, los granos, nunca entendí eso. Mi comida era normal. La máquina me seco, era una uva pasa. Llegaba de diálisis y me que- ría comer el mundo entero, pero no engordaba. Decía "para que una dieta, uno se tiene que alimentar bien". Durante la HD me cuidaba en la sal y me acostumbre a comer bajito de sal. Con las dietas no estoy de acuerdo, cuando tenía control con nutrición, decía "ellos de que quieren que uno se alimente", dicen coma yogurt deslactosado, quesos y leches caras... Vivía con mucha sed y le prohíben a uno tomar líquido y acá con semejante calor, fue terrible, sentía impotencia. ( $P-V G)$

La máquina de HD: produce miedo, sin embargo es una posibilidad de vida. Reconocen la utilidad y el funcionamiento de la diálisis; así como la presencia de la máquina de diálisis que les permite seguir vivos, a pesar de la enfermedad, la máquina es percibida como "salva vidas". El tratamiento es un proceso al que deben adaptarse, asisten al encuentro con la máquina de manera interdiaria tratando de suplir una función que el cuerpo ha perdido. Se sienten agradecidos con "ella" por prolongar su vida y darles la posibilidad de compartir con su familia un día más.

La diálisis es para limpiar la sangre, sacar el agua y toxinas que no sirven en el organismo. Un avance tecnológico muy bueno, cuantas personas nos hubiéramos muerto donde no existiera. Dios nos está dando una oportunidad, así sea con una máquina estamos prolongando la vida unos días más y compartiendo con la familia. $(P-E P)$

La máquina una "lavadora" cruel que los descompensa, al devolverle la sangre sienten que caen en un abismo, es una experiencia traumática. Así mismo, les produce miedo, sienten que los acerca a la muerte, en especial cuando se va la luz, los pitos emitidos por la emergencia los preocupa, y esto se agrava al ver el personal de Enfermería devolverle la sangre de la persona enferma. Sin embargo, con el tiempo aprenden a conocerla y reconocen la importancia que ella representa para su vida.

Se siente uno enchufado en una lavadora. La máquina es cruel, una opción de vida. Pero cuesta, sobre todo cuando va terminando uno la diálisis y cogen la sangre y la regresan otra vez, es duro para el cuerpo o cuando se sube la tensión, uno siente que cae en un abismo, me agarraba de la silla, sentía que me iba. Una sensación traumática, la máquina nunca deja de pitar, cuando pita hay que desenchufarlo. (P-MAA)

\section{La experiencia de los cuidadores en el tratamiento dialítico se describe a continuación:}


Los cuidadores familiares se sienten atados por la enfermedad y el tratamiento. Deben compartir el tiempo en que las personas están en tratamiento y estar pendientes de sus necesidades; se sienten amarrados y se les dificulta viajar; así como, realizar sus actividades laborales, sociales y de ocio, sin restricciones.

Poníamos la alarma para madrugar, entre más temprano llegara, más temprano la desconectaban, se afanaba, le daba estrés estar allá metida. En Bogotá, le hacían la HD durante tres horas, cuando se vino a Neiva le dejaron tres horas y media de tratamiento. y el estrés era estar tanto tiempo allá metida. Están atados a esa máquina. Ella se vino a vivir a Neiva y no tenía familia, pasar un puente en Bogotá era difícil, tenía que fallar a la HD. (C-GA)

Los cuidadores reconocen la importancia de la dieta para la enfermedad y el tratamiento. Los cuidadores en sus discursos identifican la dureza de la dieta, asociado a los cambios en la alimentación, hacer la comida aparte, darle alimentos diferentes y dializarlos; esto endurece la mirada de ellos en torno a la alimentación. Aprenden a manejarla por una "hojas" que le dan en la Unidad Renal. Por otro lado, no se comprometen en el control en la ingesta de líquidos, teniendo en cuenta que las personas enfermas deben ser conscientes que el consumo de líquidos desmedido los hace sentir mal y los induce a llegar a diálisis con exceso de peso.

La dieta es dura, tocaba hacerle la comida aparte. Siempre he trabajado y la comida de alguna manera tenía que resultar. LA comida para él era diferente a la de nosotros. Tenía que darle cosas que no fueran malas para él, como las que tenían mucho potasio. No tuvimos cita con la nutricionista, me dieron unas hojas, aprendí que debía comer. El consumo de líquidos, él lo controlaba, era consciente que si se sentía mal era él, toda la vida ha tomado harto líquido, Ilegaba con mucho peso a diálisis. (C-ANP)

\section{El significado del tratamiento para los profesio- nales de Enfermería y las estrategias para ayudar a} afrontar el tratamiento se describen a continuación.

En el cuidado de Enfermería, se reconoce la dureza de la HD. La HD, es descrita por la enfermeras como un tratamiento duro para la persona enferma, les restringe la posibilidad de viajar, trabajar y continuar con su vida social. Pero al mismo tiempo consideran que les da la oportunidad de continuar con su vida. Según estas, los pacientes tienen diversas representaciones del tratamiento: la dureza, el sufrimiento por las punciones de la fístula arteriovenosa, la angustia por la sangre que sale del cuerpo, estar atado a la máquina y reconocerlo como un acompañante para el resto de sus vidas.

Al principio la diálisis es fuerte, sufren por las punciones, el aumento de la tensión arterial, el vómito y la hipotensión arterial, se dan cuenta que no va a tener una vida fácil. El paciente que estaba bien hasta hace dos o tres días y llega al hospital con una encefalopatía urémica, ni se percatan de eso y, al empezar la diálisis ven la mejoría. Cuando están más conscientes y les explica que el tratamiento no es transitorio, es duro y si uno no hace un buen trabajo de diez, alcanzan a desertar dos personas. (E-VF)

Los profesionales de Enfermería, tiene la tarea de evitar los "chichotes" de la fístula arteriovenosa. En sus descripciones identifican como causa de las dilataciones de la fístula, la mala práctica en las punciones, el hecho de no rotar el sitio lleva a la formación de los "chichotes" o "culebras" que atemorizan. Estas dilataciones afectan la parte estética y las personas la ocultan de las mirada de los otros por temor a sentirse estigmatizadas. Experimentan el rechazo por la enfermedad. Una de las tareas de los profesionales de Enfermería es: educación del cuidado y conservación de la fistula.

Los chichotes de las fístulas se hacen si no se rota el sitio, no se rota porque les duele menos. Es cuestión de enseñanza, para uno como enfermera es fácil chuzar (puncionar) en el mismo sitio y esa es la peor práctica. Además es cruel la imprudencia de las personas, "Usted porque tiene esos chichotes, ¿se está dializando?" (E-DLM)

Los miran raro, extraño, inclusive la misma familia, algunos dicen "pobrecita yo con esos chichotes o me pasa corriente", el rechazo es por esa parte y las mujeres dicen "no me puedo poner si no blusa manga larga, porque voy para el centro y me comienzan a mirar raro". $(E-L M C)$

El catéter peritoneal, las dificultades para aceptarlo en el tratamiento. Las enfermeras describen la dificultad con el catéter peritoneal para la persona enferma, sienten que se afecta su imagen corporal por su presencia. Sin duda, los niños, los jóvenes y los adultos jóvenes tienen mayor dificultad de aceptar su presencia en comparación con el adulto mayor. Adicionalmente, provoca preocupación las relaciones sexuales con sus parejas, e inquietud en los hombres ante la posibilidad de presentar impotencia por su presencia. 
El catéter peritoneal es difícil para el paciente, porque va a sobresalir en ellos, para ellos es una manguera, pistola. Es más difícil para el adulto joven y el niño, qué para el anciano, a ellos le da lo mismo tenerlo o no. Usted puede ver a un sin número de pacientes haciendo artimañas para que no se les note mucho, es tenaz verse eso. (E-VF)

Uno les dice que el catéter no los afecta, la inquietud es "Mi esposa me va a rechazar por esa manguera colgando". El hombre dice "no me lo pongo por la peritonitis y porque no puedo conquistar". (E-LMC)

La dieta, un dilema para Profesionales de Enfermería. Uno de los grandes problemas de las personas es el seguimiento y adherencia a la dieta, parte del problema reside en el hecho de que al hacer estas prescripciones no se valoran las costumbres y los aspectos socioeconómicos, factores que influyen en el seguimiento o no de la dieta ordenada. Se produce un rechazo a la dieta por parte de la persona enferma, por la percepción que ellos tienen de "morirse de hambre" al seguir las indicaciones nutricionales. En esta situación, no solo existe la dificultad con los alimentos si no también con las restricciones en el consumo de agua y sal, que conduce a los enfermos a no seguir la dieta y comer los alimentos como "normalmente" lo hacían.

Las nutricionistas les prohíben todo, no preguntan cuál es la base de su comida y ellos solo tiene plátano, yuca, arroz y papa. Les decía dígale a la nutricionista que es lo que come, para que les hagan la dieta. No prohibirles todo porque se van a desnutrir, ellos dicen "si hago caso a la nutricionista me muero de hambre". La familia se ha ido y tienen que comer lo que haya. (E-DLM)

\section{Discusión}

La HD produce una ruptura en su estilo de vida, llevándolos a adaptarse a una situación que impone nuevas reglas $y$, en muchas ocasiones impide la realización de actividades de la vida diaria, según Campos y Turato ${ }^{10}$ esto se asocia al hecho de sentirse limitados por la enfermedad y el tratamiento. En este mismo sentido, Cruz et $\mathrm{al}^{2}$ consideran que estos viven una situación paradójica, por un lado cumplir con los cuidados y prescripciones para mantener el equilibrio del organismo, por otro lado, viven la aparición de "desequilibrios" de orden psicológico: estrés, agresividad y depresión.

Las personas con ERC describen el tratamiento como un evento angustiante y que los enfrenta de manera constante con la muerte. De igual manera, Campos et $\mathrm{al}^{11}$, reconocen que el tratamiento pone en evidencia elementos que representan la enfermedad, la esperanza y el cansancio, los cuales coexisten con las dificultades que tienen que aceptar, la rutina del tratamiento y la necesidad de sobrevivir. Niu y Liu ${ }^{12}$, describen la angustia y depresión, asociada a la punción, la apariencia del tratamiento y los efectos adversos; que los lleva a experimentar ansiedad y preocupación por el futuro.

De igual manera, las enfermeras reconocen la dureza del tratamiento asociada principalmente a las punciones, la pérdida sanguínea, la disminución del volumen de líquidos y al tratamiento mismo. Con referencia a lo anterior, Sciberras y Scerri ${ }^{13}$ consideran que este grupo de personas, valoran el rol del personal de salud, no solo cuando aclaran aspectos relacionados con el funcionamiento del "riñón y la máquina", sino también cuando las ayudan en la resolución de problemas de la vida personal y privada.

La presencia de la fistula arteriovenosa, es atemorizante y desagradable, tanto para la persona enferma, como para quienes comparten su vida. Cho y Shin ${ }^{5}$, concuerdan que esta produce cambios en la apariencia corporal, la presencia de las "montañitas" en el brazo es objeto de la mirada del otro, sintiéndose avergonzados del cuerpo deformado. Ramírez et al. ${ }^{14}$ y Mesa et al. ${ }^{15}$, ratifican como estas modificaciones corporales producen disminución del autoestima y cambios en la autoimagen corporal, que hacen variar la vestimenta para ocultarla, aspecto que impacta la cotidianidad de las personas en esta condición y afecta las interacciones sociales, generando altos niveles de estrés.

Sin duda, los profesionales de Enfermería son responsables de prevenir las dilataciones del acceso. Brinda educación constante en la sala entorno a las prácticas de cuidado, así como también, resuelve dudas y falsas interpretaciones en relación a la fístula arteriovenosa. Rotan el sitio de punción para prevenir las dilataciones, situación que no es bien recibido por las personas por el dolor en los nuevos sitios de punción. Enfermería debe abordar esta situación desde diversos frentes: la prevención de su formación por una práctica inadecua$\mathrm{da}$, reforzar conductas de cuidado del acceso vascular, ayudar a fortalecer la autoimagen y el autoconcepto, buscar el apoyo de la familia y ayudar en el manejo de las dilataciones cuando estas se producen.

Otro aspecto relevante es la presencia del catéter peritoneal que produce incomodidad y miedo, una forma 
de deformar el cuerpo, la "manguera" que avergüenza, es ocultada y protegida. Si bien es cierto, el curso imprevisible de la enfermedad, está acompañado de la inseguridad y el miedo, la enfermedad produce pérdida del propio cuerpo; en ese mismo sentido Sadala et al. ${ }^{16}$, describen como estos pasan a tener apéndices en él, se encuentran unidos a objetos (el catéter en el abdomen) y equipos (la máquina de diálisis), el líquido de diálisis introducido durante horas y horas, la deformación del cuerpo y presencia constante de dolor e incomodidad.

Las descripciones de las enfermeras concuerdan con la preocupación de las personas enfermas en relación a los cambios en el cuerpo ocasionado por el catéter peritoneal. En concordancia Shubayra ${ }^{17}$, considera que una de las grandes dificultades en las unidades de DP, es la comunicación con el personal de salud, por consiguiente, son necesarias estrategias de comunicación y educación, que favorezcan una educación eficiente y efectiva, para ayudar a las personas a disminuir el miedo, la ansiedad y aprender a manejar la enfermedad y el tratamiento de tal manera que mejore su calidad de vida.

En este estudio emerge la dieta, un componente importante en el tratamiento asociado con las restricciones, las modificaciones y su cumplimiento. El perder peso, se asocia a la dieta que los "mata de hambre" aspecto que influye para abandonarla de manera definitiva o seguirla parcialmente, siendo descrita como "imposibles de seguir". Hong et al. ${ }^{18}$, en su estudio reportan como las personas asumen una actitud sumisa hacia las restricciones dietéticas y de líquidos, intensificando los sentimientos de angustia y frustración, que el tratamiento impone en la vida. Kelly et al. ${ }^{19}$, describen que las personas se sienten "desanimadas" y confundidas por falta de consejos dietéticos específicos con enfoque individualizado. Estos autores agregan, como se sienten preparados y reciben poco o ningún apoyo en relación a cómo deben implementar los cambios.

Conviene destacar que la sed aparece como una constante que impide seguir las restricciones hídricas. "EI cuerpo lleno de agua", los obliga a aumentar el consumo de líquidos, convirtiéndose en un círculo vicioso que no termina, a pesar de los esfuerzos del equipo de salud por reforzar las conductas que impidan continuar con los excesos desmedidos a los que someten el cuerpo.

En las voces de los cuidadores aparece el tema de la dieta, mientras estos asumen el papel de ayudarlos a cumplirla, las personas enfermas las rechazan y no cumplen con las recomendaciones. La pareja los apoya ajustándose a la dieta de la persona enferma para ayudarla en la adherencia, otros les recuerdan constantemente los cuidados con la alimentación o preparan la comida aparte del resto de la familia siguiendo las recomendaciones de la nutricionista. Ahora bien, involucrar a los miembros de la familia es relevante porque les ayudan a regularla. En este mismo sentido, para Kelly et al. ${ }^{19}$, la dieta es una "cosa de dos personas", donde se debe incluir a quién cocina y a la persona con ERC.

Algo semejante ocurre con los profesionales de Enfermería, quienes luchan con las personas en tratamiento por la falta de adherencia a la dieta, la cual consideran está influenciada por la ausencia de valoración de las costumbres y las dificultades socioeconómicas al diseñar la formula nutricional, que afectan la gran mayoría de las personas con ERC. Ante este panorama ellas reconocen la dureza de la enfermedad, que produce demandas difíciles de acatar por las personas enfermas, llevándolos a vivir en la sala, las múltiples complicaciones que padecen por los excesos cometidos y que termina por afectar el organismo y amenazar la vida.

En concordancia con los hallazgos de este estudio, Meuleman et al. ${ }^{20}$ reconocen que los profesionales de la salud deben identificar las barreras que afectan la adherencia a la dieta e implementar estrategias orientadas a lograr cambios en el comportamiento de las personas, es necesario reconocer que el seguimiento a la dieta, el cumplimiento a las restricciones hídricas y la eliminación de la sal, no puede limitarse a una estrategia empleada en un solo momento del tratamiento. Por el contrario, se debe realizar una evaluación continua de la persona, la familia y/o el cuidador, con el fin de diseñar estrategias que refuercen las conductas positivas y corrijan aquellas consideradas negativas, para ayudarlas a asumir el control de su vida, su destino y la responsabilidad hacia ellos mismos.

Finalmente, se aborda la máquina de HD una "lavadora" cruel, que exprime, debilita y produce deterioro del cuerpo como consecuencia de su funcionamiento. Su significado está asociado al temor, el miedo y la desesperación; una amenaza constante de la muerte que los enfrenta a la finitud de su vida. Además, Cho y $\mathrm{Shin}^{5}$, reconocen que vivir con esta es estar bajo la sombra de la muerte, las personas experimentan impotencia y ansiedad, al mismo tiempo que están dispuestas a enfrentar la muerte que puede llegar en cualquier momento, durante el tratamiento. 
Además, los participantes en la investigación describen la máquina de HD como una tecnología que les permite vivir y compartir con su familia un día más. Para Morales y Castillo ${ }^{21}$, esta produce frustración por estar pegados a ella, situación descrita como un tiempo desperdiciado, al mismo tiempo que es percibida como salvadora de vidas. Polikandrioti et al. ${ }^{22}$, concuerdan que entorno a la máquina de diálisis y el acceso vascular las personas han construido sus propios significados, el acceso vascular como un recordatorio visual de la enfermedad y la máquina completamente necesaria para la supervivencia.

A partir de los resultados se puede concluir, que la HD asume la identidad de ser dura, un tratamiento que ayuda a sobrevivir, pero con efectos fuertes que amenaza la vida de las personas con ERC, un vínculo con la vida y la muerte, sentimientos compartidos con las enfermeras que intentan ayudarlos a sobrellevar esta experiencia devastadora. El cuerpo deformado por la fistula y el catéter peritoneal, es ocultado, percibido como una interferencia en sus relaciones sociales y de pareja, en la cual los profesionales de Enfermería asume un papel importante al evitar las deformidades así como ayudarlos en el proceso de aceptación a estos "apéndices" y "montañas" que aparecen inesperadamente.

La dieta significa para las personas morirse de hambre y los lleva a no seguirla, mientras los cuidadores y las enfermeras luchan por ayudarlos en la adherencia y de esta forma evitar las complicaciones y mejorar la calidad de vida en el tratamiento. El temor a la "Máquina", no es otra cosa que el miedo al deterioro, las complicaciones, la proximidad de la muerte, el trapiche que exprime, mata y al mismo tiempo salva vidas; una extensión del cuerpo a la que simultáneamente se teme y se ama, una "cosa" con significado en su realidad circundante.

\section{Agradecimientos}

Agradezco a las personas con ERC trasplantadas que participaron en este proyecto, sus cuidadores y el personal de Enfermería. Así mismo agradezco a la Unidad de trasplante Surcolombiano por su apoyo con las bases de datos.
Recibido: 16-03-19

Revisado: 20-05-19

Modificado: 15-06-19

Aceptado: 01-07-19

\section{Bibliografía}

1. Ayar Y, Ersoy A, Ocakoglu G, Yildiz A, Oruc A, Soyak $\mathrm{H}$, et al. Risk Factors Affecting Graft and Patient Survivals After Transplantation From Deceased Donors in a Developing Country: A Single-Center Experience. Transplant Proc 2017;49(2):270-7.

2. Cruz V, Tagliamento G, Wanderbroocke AC. A manutenção da vida laboral por doentes renais crônicos em tratamento de hemodiálise: uma análise dos significados do trabalho. Saúde e Soc. 2016 Dec;25(4):1050-63.

3. Bergjan M, Schaepe C. Educational strategies and challenges in peritoneal dialysis: $A$ qualitative study of renal nurses' experiences. J Clin Nurs. 2016;25(11-12):1729-39.

4. Gill P. Stressors and coping mechanisms in live-related renal transplantation. J Clin Nurs 2012;21(1112):1622-31.

5. Cho M, Shin G. Gender-based experiences on the survival of chronic renal failure patients under hemodialysis for more than 20 years. Appl Nurs Res. 2016;32:262-8.

6. Schaepe C, Bergjan M. Educational interventions in peritoneal dialysis: A narrative review of the literature. Int J Nurs Stud. 2015;52(4):882-98.

7. Van Manen M. Phenomenology of practice: meaning-giving methods in phenomenological research and writing. $1^{\text {a }}$ ed. Walnut Creek: Routledge; 2014. p. 411.

8. Patton M. Qualitative evaluation methods and Research Methods. 3rd ed. London: Sage; 2002. p. 806. 
9. Lincoln $Y$, Guba E. Naturalistic inquiry. Naturalistic inquiry. $1^{\text {a }}$ ed. Beverly Hills: Sage; 1985. p. 416.

10. Campos C, Turato E. Hemodialysis treatment as perceived by the renal patient: clinical qualitative study. Rev Bras Enferm. 2010;63(5):799-805.

11. Campos C, Mantovani F, Nascimento M, Cassi C. Social representations of illness among people with chronic kidney disease. Rev Gaucha Enferm. 2015;36(2):106-12.

12. Niu H, Liu J. The psychological trajectory from diagnosis to approaching end of life in patients undergoing hemodialysis in China: A qualitative study. Int J Nurs Sci. 2017;4(1):29-33.

13. Sciberras A, Scerri J. Facilitators and barriers to treatment with haemodialysis among persons in mid-adulthood: An interpretative phenomenological analysis. Scand J Caring Sci. 2017;31(4):695-701.

14. Ramírez M, Ferrada M, Silva A, Courtin A, Malabrigo $P$. Exploring sexuality in women undergoing dialysis: A qualitative approach. Rev Nefrol Dial y Traspl. 2018;38(1):35-42.

15. Mesa L, Carrillo A, Castiblanco R, Reina L, Ávila $T$. Renal replacement therapy: A meta-study and synthesis of qualitative evidence. Aquichan. 2017; 17(3):328-52.

16. Sadala M, Bruzos G, Pereira E, Bucuvic E. Patients' experiences of peritoneal dialysis at home: a phenomenological approach. Rev Lat Am Enfermagem. 2012;20(1):68-75.
17. Shubayra A. Continuous ambulatory peritoneal dialysis: nurses' experiences of teaching patients. Saudi J Kidney Dis Transpl. 2015;26(2):309-13.

18. Hong L, Wang W, Chan E, Mohamed F, Chen H. Dietary and fluid restriction perceptions of patients undergoing haemodialysis: an exploratory study. J Clin Nurs. 2017;26(21-22):3664-76.

19. Kelly J, Campbell K, Hoffmann T, Reidlinger D. Patient Experiences of Dietary Management in Chronic Kidney Disease: A Focus Group Study. J Ren Nutr. 2018;28(6):393-402.

20. Meuleman $Y$, Brinke $L$, Kwakernaak $A$, Vogt $L$, Rotmans J, Bos W, et al. Perceived Barriers and Support Strategies for Reducing Sodium Intake in Patients with Chronic Kidney Disease: a Qualitative Study. Int J Behav Med. 2015;22(4):530-9.

21. Morales L, Castillo E. Vivencias de los adolescentes en diálisis: una vida con múltiples pérdidas pero con esperanza. Colomb Med. 2007;38(2):44-52.

22. Polikandrioti M, Koutelekos I, Gerogianni G, Stefanidou S, Kyriakopoulos V, Floraki E, et al. Factors Associated with Hemodialysis Machine Dependency. Med Arch. 2017;71(2):122-7.

Este artículo se distribuye bajo una Licencia Creative Commons Atribución-NoComercial 4.0 Internacional. https://creativecommons.org/licenses/by-nc/4.0/

Open Access (C) (1) (8) 